Hautarzt $2021 \cdot 72: 453-455$

https://doi.org/10.1007/s00105-020-04695-z

Online publiziert: 26 . September 2020

(c) Der/die Autor(en) 2020

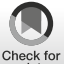

Christina Martha Vallant · Daisy Kopera

Klinik für Dermatologie, Medizinische Universität Graz, Graz, Österreich

\title{
Schmerzhafte Knoten am Oberarm
}

\section{Klinischer Befund}

Am linken Oberarm finden sich 3 etwa $5-10 \mathrm{~cm}$ voneinander entfernte Knoten. Die unmittelbare Umgebung ist sehr diskret gerötet. Nach Ablösen einer der Krusten zeigt sich im Zentrum des Knotens ein derber gelblicher Pfropf (• Abb. 1). Beim Versuch, den Pfropf zu exprimieren, klagt der Patient über starke Schmerzen, sodass ein Lokalanästhetikum zur Anwendung kommt.

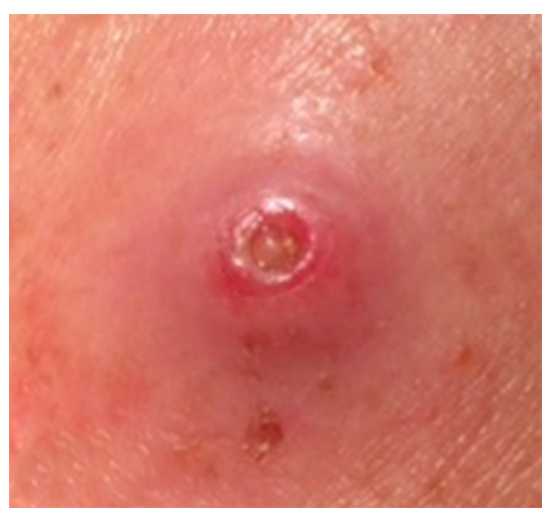

Abb. $1 \Delta$ Knoten mit darin befindlicher Made mit Atmungsöffnung am Hinterleib

\section{Wie lautet lhre Diagnose?}

\section{Weiteres Procedere/Therapie}

Während der Applikation des Lokalanästhetikums wölbt sich der gelbliche Pfropf immer weiter vor, und es entpuppt sich eine kleinfingerdicke Made (• Abb. 2a). Mit weiterem Druck durch das subkutan injizierte Lokalanästhetikum von unten her kann mithilfe einer Pinzette eine $35 \mathrm{~mm}$ lange Made entwickelt werden (- Abb. 2b). Gleichartig werden die beiden restlichen Läsionen behandelt, auch daraus lassen sich ebensolche Parasiten ziehen, die nach Recherche eindeutig als Larven/Maden einer Dasselfliege identifiziert werden können (• Abb. 3).

\section{Klinische Differenzialdiagnosen}

Differenzialdiagnostisch (in alphabetischer Reihenfolge) müssen in Erwägung gezogen werden:

- Arthropodenreaktion,

- Epidermiszysten,
- Furunkulosis,

- Leishmaniose,

- Malignome: Merkel-Zell-Karzinom, kutanes Lymphom, Hautmetastase,

- Pyoderma,

- Sporotrichose,

- Xanthogranulom.

\section{Definition}

Während die afrikanische Tumbufliege (auch Mangofliege, Cordylobia anthropophaga), ein Ektoparasit aus der Gattung Cordylobiae, ihre Eier auf feuchter Unterlage, auch z. B. auf verschwitzter Kleidung ablegt und die schlüpfenden Larven (= Maden) sich aktiv in die Haut bohren, legt die südamerikanische Dasselfliege (Dermatobia hominis) aus der Familie der Oestridae ihre Eier auf ein blutsaugendes Insekt, üblicherweise auf Stechmücken oder Zecken, damit diese die Eier auf die Haut von Säugetieren übertragen. Man nennt das Phoresie

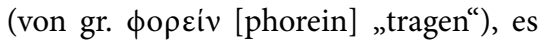
handelt sich um eine „vorübergehende Transportgesellschaft“. Dort entwickeln sie sich aufgrund der Körpertemperatur des Wirts weiter, und die Larven gelangen durch kleinste oberflächliche Wunden oder Haarfollikel in die Haut, oder die Fliegen legen Eier direkt auf Wunden $[1,2]$. Charakteristische Widerhäkchen erleichtern das Eindringen. Die Larven ernähren sich in der Haut von Exsudaten, machen 3 Larvenstadien durch und verlassen dann den Wirt, um sich in der Erde $\mathrm{zu}$ verpuppen und zur Fliege weiterzuentwickeln [1,3]. Mitunter verursachen die heranwachsenden Larven nicht nur ein Kribbeln (durch die sich bewegenden Widerhäkchen), sondern auch furunkuloide Entzündungsherde, auch Dermalmyiasis genannt [4]

Begleitend kann es zu einer bakteriellen, häufig Staphylokokken-induzierten Superinfektion mit Fieber und regionärer Lymphadenopathie kommen, wo 

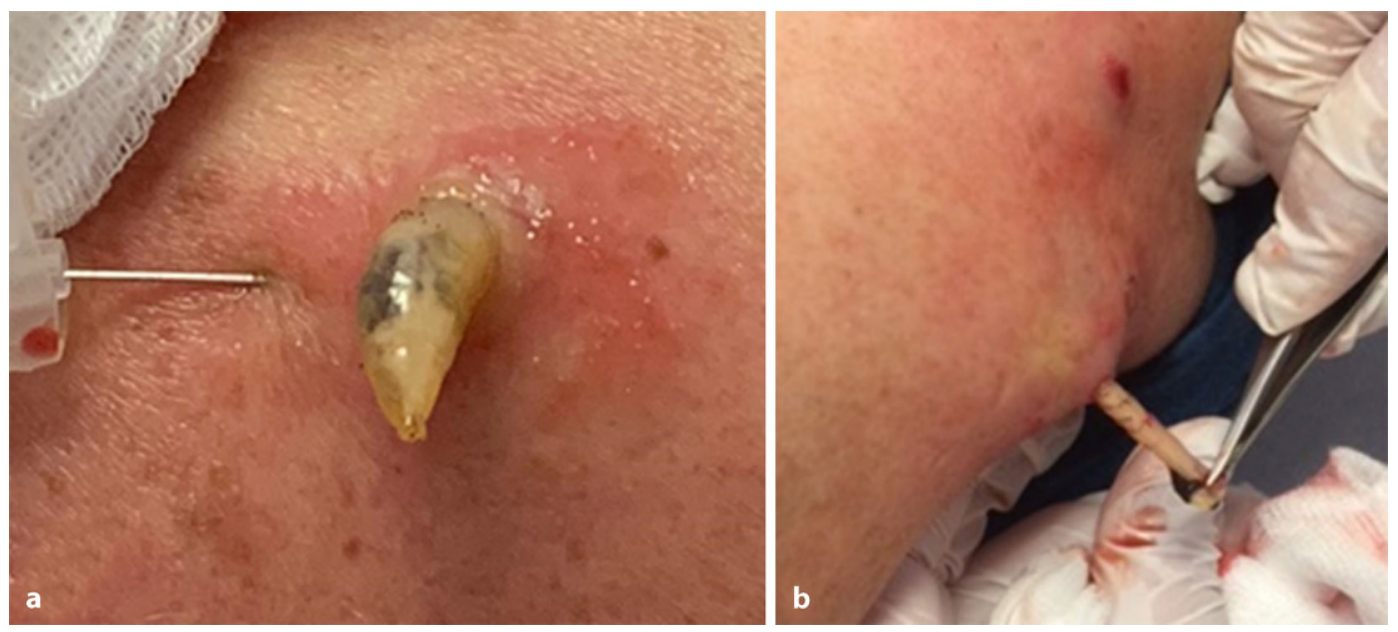

Abb. $2 \triangleleft$ Applikation des Lokalanästhetikums subkutan unter den furunkuloiden Knoten und hervortretende Made (a) Herausziehen der Made aus der Haut des Wirts (b)

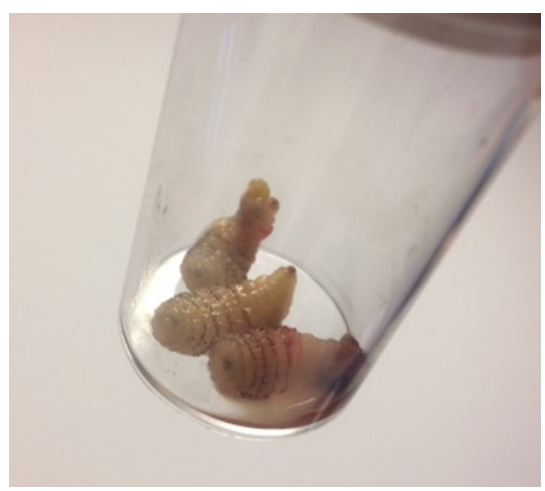

Abb. $3 \Delta$ Drei Dasselfliegenlarven (= Maden) nach Exkochleation im Reagenzglas

der Einsatz eines Antibiotikums erwägenswert ist. Die Therapie der Wahl ist die lokale Entfernung der Larve. In der Literatur werden neben Inzision der Höhle auch andere, kuriose therapeutische Ansätze wie die Auflage von Speck [5] beschrieben. Durch den Okklusionsverband mit einer Scheibe Schweinespeck wird die am Hinterleib sitzende Atemöffnung der Made luftdicht abgedeckt. Um an Luft zu kommen, wandert sie durch den Speck an die Oberfläche und verlässt so atraumatisch die Haut des temporären Wirts. In unserem Fall gelang es durch subkutane Unterspritzung der Larvenhöhlen mit einem Lokalanästhetikum (• Abb. 2a), sie durch Druck von unten durch die als Atemöffnung dienende Hautpore atraumatisch und in toto $\mathrm{zu}$ bergen (• Abb. 2b).

\section{》) Diagnose: Furunkuloide kutane Myiasis}

\section{Diskussion}

Denkt man an die steigende Inzidenz der Skabies, so sind Parasitosen in der westlichen, zivilisierten Welt gar nicht so selten. Die Läsionen die durch Larven (Maden) tropischer Fliegen verursacht werden, kommen aber relativ selten vor. Die ersten Differenzialdiagnosen, die bei Betrachtung derartiger Hautläsionen in Erwägung gezogen werden, gehen zumeist nicht in diese Richtung. Es sind jedoch die einfachen Dinge, die uns beim Anblick einer Hautveränderung in den Sinn kommen sollten [6,7]. Ganzklar war hier die Anamnese unvollständig, denn im Nachhinein erzählte der Patient, dass er vor etwa 5 Wochen von einer Wanderreise durch Costa Rica heimgekehrt sei und dort in feuchtheißem Regenwaldklima gelegentlich auch unter eher abenteuerlichen Umständen übernachtet habe. Kontakt mit diversen Insekten sei keine Seltenheit gewesen.

\section{Fazit für die Praxis}

Es wird empfohlen, bei der Anamneseerhebung sorgsam zu sein und auch Auslandsaufenthalte bzw. Kontakt zur Natur zu erfragen.

\section{Korrespondenzadresse}

Univ. Prof. Dr. Daisy Kopera

Klinik für Dermatologie, Medizinische

Universität Graz

Auenbruggerplatz 8, 8036 Graz, Österreich daisy.kopera@medunigraz.at

Funding. Open access funding provided by Medical University of Graz.

\section{Einhaltung ethischer Richtlinien}

Interessenkonflikt. C.M. Vallant und D. Kopera geben an, dass kein Interessenkonflikt besteht.

Für diesen Beitrag wurden von den Autoren keine Studien an Menschen oder Tieren durchgeführt. Für die aufgeführten Studien gelten die jeweils dort angegebenen ethischen Richtlinien. Für Bildmaterial oder anderweitige Angaben innerhalb des Manuskripts, über die Patienten zu identifizieren sind, liegt von ihnen und/oder ihren gesetzlichen Vertretern eine schriftliche Einwilligung vor.

Open Access. Dieser Artikel wird unter der Creative Commons Namensnennung 4.0 International Lizenz veröffentlicht, welche die Nutzung, Vervielfältigung, Bearbeitung, Verbreitung und Wiedergabe in jeglichem Medium und Format erlaubt, sofern Sie den/die ursprünglichen Autor(en) und die Quelle ordnungsgemäß nennen, einen Link zur Creative Commons Lizenz beifügen und angeben, ob Änderungen vorgenommen wurden.

Die in diesem Artikel enthaltenen Bilder und sonstiges Drittmaterial unterliegen ebenfalls der genannten Creative Commons Lizenz, sofern sich aus der Abbildungslegende nichts anderes ergibt. Sofern das betreffende Material nicht unter der genannten Creative Commons Lizenz steht und die betreffende Handlung nicht nach gesetzlichen Vorschriften erlaubt ist, ist für die oben aufgeführten Weiterverwendungen des Materials die Einwilligung des jeweiligen Rechteinhabers einzuholen. 
Weitere Details zur Lizenz entnehmen Sie bitte der Lizenzinformation auf http://creativecommons.org/ licenses/by/4.0/deed.de.

\section{Literatur}

1. Calvopina M, Ortiz-Prado E, Castañeda B, Cueva I, Rodriguez-Hidalgo R, Cooper PJ, Karunaweera ND (2020) Human myiasis in Ecuador. PLoS Negl Trop Dis 14:e7858. https://doi.org/10.1371/journal. pntd. 0007858

2. Safdar N, Young DK, Andes D (2003) Autochthonous furuncular myiasis in the United States: case report and literature review. Clin Infect Dis 36:e73-80

3. Pereira MCT, Leite VHR, Leite ACR (2001) Experimental skin lesions from larvae of the bot fly Dermatobia hominis. Med Vet Entomol 15:22-27

4. Rufli T (2005) Epizoonosen. In: Braun-Falco O, Plewig G, Wolff HH, Burgdorf WHC, Landthaler M (Hrsg) Dermatologie und Venerologie, 5. Aufl. Springer, Heidelberg, S292-310

5. Brewer TF, Wilson ME, Gonzalez E, Felsenstein D (1993) Bacon therapy and furuncular myiasis. J Am Med Assoc 270:2087-2088

6. Solomon M, Lachish T, Schwartz E (2016) Cutaneous myiasis. Curr Infect Dis Rep 18:28. https://doi.org/10.1007/s11908-016-0537-6

7. Oliva E, Bargiggia G, Quinzan G, Lanza $P$, Farina C (2020) Furuncular myiasis in Italian traveler returning from Kenya. J Infect Dev Ctries 14:114-116. https://doi.org/10.3855/jidc. 11560

\section{Hier steht eine Anzeige.}

\section{Springer}

